\title{
Epidemiologia: ELA no Mundo
}

\author{
Epidemiology: ALS in World
}

\section{Roberto Dias Batista Pereira}

Vice-Presidente da Associação Brasileira de Esclerose Lateral Amiotrófica - ABRELA. Mestrando em Ciências da Saúde pela Escola Paulista de Medicina / Universidade Federal de São Paulo - UNIFESP.

Acredita-se que a incidência da Esclerose Lateral Amiotrófica (ELA) seja a mesma em todo o mundo, contudo, não há um estudo comparativo bem conduzido da ELA entre populações diferentes, grupos étnicos ou área geográfica definida fora da Europa e da América do Norte. No mundo a prevalência (número de casos existentes) é de 3 - 8 casos por 100.000 habitantes, e tem uma incidência por ano (número de novos casos) de 2/100.000. Entretanto, estudos isolados mostram que existem variações. Algumas seletas regiões no mundo chamam a atenção quanto à taxa de incidência por serem mais elevada, como na ilha de Guam, em Nova Guiné ocidental, e na península de Kii no Japão.

Nos Estados Unidos a epidemiologia é bem conhecida devido ao grande número de trabalhos científicos realizados. Sabe-se que 5.000 novos casos são diagnosticados com ELA a cada ano, correspondendo, aproximadamente, a 13 novos casos por dia, incidência de 2/100.00. A metade de todos os pacientes afetados vivem pelo menos 3 anos ou mais após o diagnóstico. Em torno de $20 \%$ vivem 5 anos ou mais, e até $10 \%$ sobrevivem mais de 10 anos. A maioria encontra-se entre 40 e 70 anos. Acomete geralmente mais homens do que mulheres, embora, trabalhos mais recentes mostram que ambos os sexos estão sendo afetados de forma semelhante.

Relatos do aumento no número de casos de ELA em mulheres podem estar relacionados a melhor identificação da população com estudos já realizados, a efeitos de uma exposição ambiental recente não identificada, e/ou mudanças no estilo de vida, fazendo com que estas sejam expostas à potenciais tóxicos, associando historicamente com os homens (fumo, exposições ocupacionais).

Referente aos países europeus foi realizado uma pesquisa a qual foram selecionados 26 estudos europeus e 28 não-europeus, apresentando uma incidência de ELA variando de 1,7(Itália) a 2,4 (Finlândia média) por 100.000/ano na Europa e de 0,31 (China) a 1,98 
(Estônia) por 100.000/ano fora da Europa. Outros países e regiões com estudos de ELA no mundo (Tabela 1).

Tabela 1. Países países e regiões com estudos de ELA.

\begin{tabular}{lccc}
\hline Regi ÕeS & $\begin{array}{c}\text { Incidência } \\
\text { (por 100.000 habitantes) }\end{array}$ & $\begin{array}{c}\text { Prevalência } \\
\text { (por 100.000 habitantes) }\end{array}$ & $\begin{array}{c}\text { Ano } \\
\text { Publicação }\end{array}$ \\
\hline Guam & 3,9 & & 2004 \\
Líbia & 3,4 & & 2005 \\
Austrália & 2,9 & 4,7 & 2005 \\
Irlanda & 2,8 & $11,31 *$ Wakayama & 1999 \\
Japão * & 2,5 & & 2005 \\
Finlândia & 2,4 & $3-8$ & 2006 \\
EUA * & 2,0 & 6,7 & 2004 \\
Canadá * & 2,0 & & 2005 \\
Estônia & 1,9 & & 2006 \\
Noruega & 1,9 & & 2005 \\
Inglaterra & 1,7 & 4,03 & 2001 \\
Itália * & 1,7 & & 2005 \\
Brasil & 1,5 & & 2098 \\
França * & 1,5 & & 2000 \\
Grécia & 1,3 & & 2005 \\
Polônia & 0,8 & & 2005 \\
Chile & 0,5 & & \\
México & 0,4 & & \\
China & 0,3 & & \\
\hline \hline & & & \\
\hline
\end{tabular}

* Média da incidência dos trabalhos realizados no país.

Nos EUA dois trabalhos de incidência e três em mortalidade sugerem um risco maior para ELA entre brancos quando comparados aos brancos latino-americanos e aos afroamericanos, entretanto, 0,61 maior em negros do que em brancos, 2,08 maior em espanos e asiáticos em relação a outros brancos.

Outros fatores chamam a atenção, como a exposição ao choque elétrico severo, atividade física extenuante, soldados que serviram na primeira guerra do Golfo, e jogadores de futebol profissional (soccer). 
Um estudo recente realizado na Irlanda discutindo a relação da ELA com a atividade física extenuante, identificou nas maratonas na cidade de Dublin (1980-2002) maratonistas irlandeses com diagnóstico de ELA. Num total de 59.604 maratonistas, 14.443 eram irlandeses, destes 661 eram acometidos com ELA, diagnosticados entre os anos de 1993 2002. Esse trabalho, ainda em continuidade, representa o primeiro estudo epidemiológico a fim de determinar o risco de ELA entre um largo "cohort" de indivíduos envolvidos em exercícios vigorosos não-profissionais.

Em relação aos veteranos da guerra do Golfo, o risco relativo de morrer de ELA é de 1,5 vezes maior que nos homens que não serviram. $\mathrm{O}$ aumento na mortalidade de ELA foi observado entre os homens que serviram o exército ou a guarda nacional $(R R=1.54)$, marinha $(R R=1.87)$, força aérea $(R R=1.54)$, e guarda costeira $(R R=2.24)$; nenhum aumento no risco foi encontrado nos homens que serviram o corpo de fuzileiros navais, embora houvesse somente 13.670 homens neste grupo. Os números mostraram que 281.874 homens que serviram as forças armadas dos EUA, 217 morreram de ELA, comparado a 63 dos 126.414 que não serviram.

Quanto aos jogadores de futebol profissional na Itália, 18 casos com ELA foram identificados dentre 7.000 jogadores. Cientistas sugerem diversas explanações, nenhuma delas com certeza. Talvez a ELA esteja relacionada ao exercício físico pesado, e conseqüentemente não relacionado particularmente ao futebol, ou talvez envolvendo o trauma principal em cabecear a bola, ou a repetição dos traumas que envolvem os membros inferiores, sejam os fatores relacionados. As drogas terapêuticas ilegais ou legais podem também estar envolvidas, e é possível que as toxinas ambientais como os fertilizantes ou os herbicidas usados nos campos de futebol tenham a sua influência.

A mortalidade mundial da ELA ainda não está bem definida, todavia, pode-se destacar os principais dados encontrados na literatura.

Nos EUA as taxas totais da mortalidade de Doença do Neurônio Motor (DNM) aumentaram de 1.25 para 1.82/100.000, representando um aumento de $46 \%$ durante o período de 30 anos (1969-1998). As taxas entre as mulheres aumentaram 60\% e continuam aumentando. As taxas entre homens cresceram 35\% durante esse período. As taxas de mortalidade entre afro-americanos e hispânicos foi aproximadamente 50\% mais baixas do que 
as taxas entre brancos não-hispânico. As taxas de mortalidade da DNM variam de 2.22 (1.89 a 2.55) no noroeste, e 1.57 (1.44 a 1.71) no sudeste.

Já na França foram analisados 9.005 mortes de ELA entre os anos de 1968 e 1982. As taxas totais de mortalidade eram 1.45/100.000 para homens e 0.90/100.000 para mulheres. Foi encontrado um aumento substancial na mortalidade de ELA sobre o tempo: as taxas ajustadas (por 100.000) no período de 1968 a 1971 eram 1.11 para homens e 0.63 para mulheres. No período 1979 a 1982, correspondem respectivamente entre 1.92 e 1.12 . O aumento foi principalmente em pacientes abaixo de 55 anos de idade e afetados na maior parte em mulheres identificadas nos primeiros anos (1968 a 1978). Nos anos mais recentes há um aumento similar em ambos os sexos.

Estudos na Noruega mostram que a mortalidade anual de ELA dobrou em um estudo, elevando-se de 1.38 a 2.54 por 100.000 habitantes no fim do estudo. Esta mortalidade aumentou na população acima de 60 anos de idade, com um pico no grupo de idade entre 80 a 84 anos. $\mathrm{O}$ aumento era maior nas mulheres do que nos homens, e a relação entre os sexos foi de 1.32. Os autores relataram que há um aumento na mortalidade de ELA na Noruega. Embora o aumento da mortalidade seja restrito à população com 65 anos ou mais, isto não pode ser explicado somente por um aumento da idade da população em geral.

A ELA sempre foi conhecida como uma doença "rara", entretanto, mundialmente chama a atenção pelo número crescente de novos casos, mesmo isolados. Sendo assim, estima-se que a incidência mundial esteja em um crescente como um todo.

\section{Referências Bibliográficas}

1. Noonan CW, White MC, Thurman D, Wong LY. Temporal and geographic variation in United States motor neuron disease mortality, 1969-1998. Neurology 2005;64:1215-1221.

2. Seljeseth YM, Vollset SE, Tysnes OB. Increasing mortality from amyotrophic lateral sclerosis in Norway? Neurology 2000;55:1262-1266.

3. Durrleman S, Alperovitch A. Increasing trend of ALS in France and elsewhere: are the changes real? Neurology, 39(6):768-773.

4. Kurtzke JF. Epidemiology of amyotrophic lateral sclerosis. Adv Neurol. 1982;36:281-302. 
5. Preux PM, Druet-Cabanac M, Couratier P, Debrock C, Truong T, Marcharia W, et al. Estimation of the amyotrophic lateral sclerosis incidence by capture-recapture method in the Limousin region of France. J Clin Epidemiol. 2000;53(10):1025-1029.

6. Beghi E. The role of epidemiology in research and care provision. Joint Opening Session 2003.

7. Epidemiology Studies Explore Potential Risk Factors for ALS. One Study: Veterans May Have Higher Risk of ALS; Another Suggests Physical Activity Not a Risk Factor, 2005.

8. Logroscino G, Beghi E, Zoccolella S, Palagano R, Fraddosio A, Simone IL, et al. Incidence of amyotrophic lateral sclerosis in southern Italy: a population based study. J Neurol Neurosurg Psychiatr 2005;76:1094-1098.

9. Elissa B. A Mind Trapped in a Body. Biology 2000, 202 Second Web Report on Serendip.

10. Kihira T, Yoshida S, Hironishi M, Miwa H, Okamato K, Kondo T. Changes in the incidence of amyotrophic lateral sclerosis in Wakayama, Japan. Amyotroph Lateral Scler Other Motor Neuron Disord. 2005;6(3):155-163.

11. Logroscino G, Beghi E, Zoccolella S, Palagano R, Fraddosio A, Simone IL, et al. Incidence of amyotrophic lateral sclerosis in southern Italy: a population based study. J Neurol Neurosurg Psychiatry. 2005;76(8):1094-1098.

12. Weisskopf MG, O'Reilly EJ, McCullough ML, Calle EE, Thun MJ, Cudkowicz M, et al. Prospective study of military service and mortality from ALS. Neurology. 2005;64(1):32-37.

13. Sejvar JJ, Holman RC, Bresee JS, Kochanek KD, Schonberger LB. Amyotrophic lateral sclerosis mortality in the United States, 1979-2001. Neuroepidemiology. 2005;25(3):144-152.

14. Argyriou AA, Polychronopoulos P, Papapetropoulos S, Ellul J, Andriopoulos I, Katsoulas G, et al. Clinical and epidemiological features of motor neuron disease in south-western Greece. Acta Neurol Scand. 2005;111(2):108-113.

15. Cronin S, Hardiman O, Traynor BJ. Motor neuron disease: Ethnic diversity in ALS - a metaanalysis. 16th Meeting of the European Neurological Society, 2006.

16. Cronin S, Hardiman O, Traynor BJ. Motor neuron disease: Risk of ALS in Irish marathon runners. 16th Meeting of the European Neurological Society, 2006. 